\title{
A genetic algorithm for the integrated berth allocation and quay crane scheduling problem in container terminals
}

\author{
Jie Ren ${ }^{1, \mathrm{a}}$, Xiao Ting Shang ${ }^{1, \mathrm{~b}}$ and Jin Xin $\mathrm{Cao}^{2, \mathrm{c},{ }^{*}}$ \\ ${ }^{1}$ School of Mathematical Sciences, Inner Mongolia University, Hohhot, 010021, P.R. China \\ 2 Institute of Transportation Engineering, Inner Mongolia University, Hohhot, 010070, P.R. China

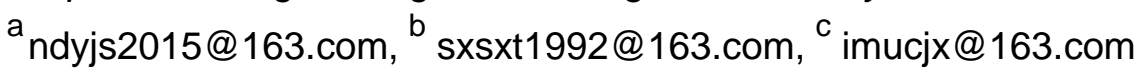 \\ ${ }^{*}$ corresponding author
}

Keywords: Discrete berth allocation problem; Quay crane scheduling; Cost balance; Integer programming model; Genetic algorithm

\begin{abstract}
Because the cost of berths and quay cranes are very high compared to the investment costs for the other facilities in container terminals, so berths and quay cranes are the most critical resources in determining the capacity of container terminals. In order to efficiently utilize berths and quay cranes, this paper studied the dynamic discrete berth allocation and quay crane scheduling problem considering the cost balance among different vessels. The objective of the proposed model is to reduce the cost of berths and quay cranes, including the handling cost, the waiting cost and the quay crane operation cost. Due to the inherent computation complexity, a genetic algorithm is designed to attain the near optimal solution. Computational experiments show that the two proposed algorithm are effective for solving the studied problem.
\end{abstract}

\section{Introduction}

On the operational level of container terminals, decision problems are typically identified as the berth allocation problem (BAP), the quay crane assignment problem (QCAP), the storage allocation problem and the yard truck scheduling problem. The core competence of container terminals is to serve vessels by discharging and charging containers. Vessel operators expect the service to be as fast as possible. Fast service operations require a careful disposition of the seaside resources, namely berths and quay cranes. So berth and quay crane are the key factors in the BAP and QCAP, whereas they are two types of scarce and expensive resources.

Quay cranes are one of the key terminal equipment used for container movement. This implies that inefficient quay crane employment could be bottleneck for fast ship handling operation. In order to reduce the effects caused by quay cranes frequent operation, we assume that cranes are transferred from one berth to another when the task at the first berth is finished. Figure 1 illustrates QCs movement across berths in a chronological time axis. It divides terminal in three berths and the length of a vessel (abscissa) represents the handling time.

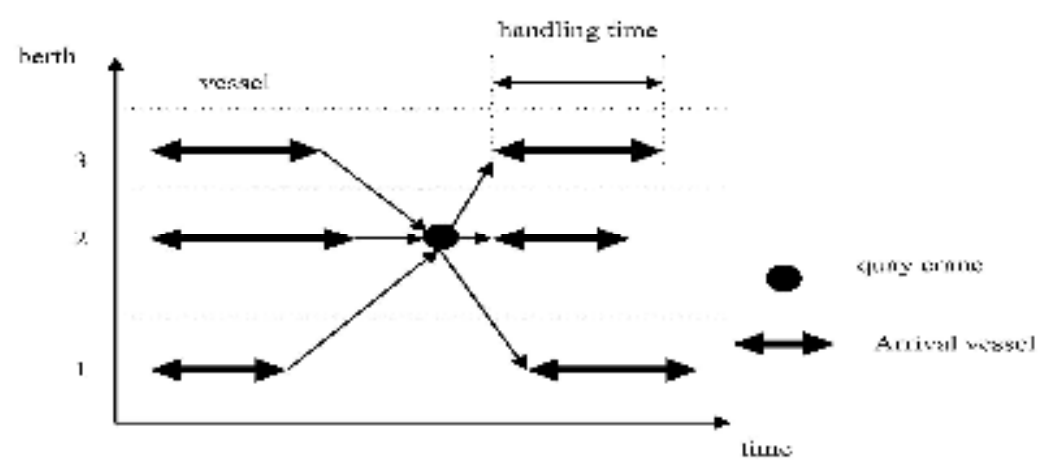

Figure 1: Berth allocation and quay crane scheduling plan 
An integrated problem to the BAP and QCAP leads to the Berth Allocation and Quay Crane Assignment Problem(B\&CAP). However, the utilization of QCs resources needs to be considered under more realistic circumstances. This issue is approached in this paper by a new model formulation for B\&CAP that considering the cost balance among different vessels. From the computational complexity point of view, the B\&CAP is complex, since BAP has been proven to be NP-hard problem. It is more difficult to deal with the B\&CAP problem. To effectively solve the problem for the large scale problem, it is necessary to develop valid solution algorithms.

In recent years, a number of researches can be found for the BAP and the QCAP in container terminals.

[1] proposed a discrete BAP inspired by the usage of more efficient terminal in Hong Kong International Terminal. [2] examined the QCAP to minimize the aggregated costs of delay with the assumptions that vessels were divided into holds and only one QC can work on a hold at a time. [3] studied the B\&CAP to minimize the total service time and the problem was solved by GA-based heuristic. [4] proposed a mixed integer linear programming model to minimize the total weighted service time of incoming vessels, and developed a meta-heuristic algorithm to obtain near-optimal solutions.

There are also a few studies which deal with the deterministic models considering the cost balance. However, it is necessary to take this part into the B\&CAP. Thus this paper studies the integrated dynamic discrete BAP and QCAP considering the cost balance. To obtain the solution of this problem, a genetic algorithm (GA) and a greedy heuristic algorithm are proposed.

\section{Model Formulation}

\section{Assumption.}

The following is assumed for the formulation of the problem:

1. Each berth can serve one vessel at most at one time.

2. There are no physical or technical restrictions such as vessel draft and water depth.

3. Vessels arrive in container terminals dynamically and the arriving times of vessels are known.

4. Container terminal will be divided by an equal distance and the length of the berth is larger than the length of any vessel to be served.

5. Once the vessel is in berth, it does not move until all tasks are completed.

6. QCs are transferred from one berth to another when the task of the precious vessel is finished.

\section{Deterministic model}

The following notations are also used in the model formulation: Input dates:

$I$ : set of berths

$J$ : set of vessels

$Q:$ total number of QCs

$K$ : set of service orders of berth

$T$ : set of periods of time

$h_{j}$ : arriving time of vessel $j \in J$

$e_{j}$ : expected leaving time of vessel $j \in J$

$s_{j}$ : expected arriving time of vessel $j \in J$

$E_{j}:$ total weight of vessel $j \in J$

$r_{j}^{\text {min }}:$ minimum number of QCs agreed to serve $j \in J$ simultaneously

$r_{j}^{\max }$ : maximum number of QCs agreed to serve $j \in J$ simultaneously

$C^{1}$ : the cost of a quay crane for $1-\mathrm{h}$

$C^{2}$ : the cost of berth for 1-h 
$C^{3}:$ the cost of waiting for $1-\mathrm{h}$

$v_{0}$ : weight of containers is discharged by one QC in an

hour $\alpha$ : interference exponet

$\beta$ : cost balance

$M$ : large constant

Decision variables:

$z_{j}:$ number of QCs allocated to vessel $j \in J$

$f_{i j k}$ : starting time of serving vessel $j \in J$ at berth $i \in I$ as the $k$ th vessel

$b_{i j k}$ : ending time of serving vessel $j \in J$ at berth $i \in I$ as the $k$ th vessel

$x_{i j k}:=1$, if vessel $j \in J$ is served at berth $i \in I$ as the $k$ th vessel, $=0$, otherwise

$v_{i j}:=1$, if vessel $j \in J$ are served at berth $i \in I,=0$, otherwise

$\theta_{q j t}:=1$, If $q$ QCs are assigned to vessel $j \in J$ at time $t \in T,=0$,otherwise

In general, once a vessel has anchored at the assigned berth, only when previous vessel has finished and a number of QCs have been ordered, vessels can start to work. The total service cost include the handling cost, the waiting cost and the QCs operation cost.

The balance of cost degree between two vessels calculated by formula (1). We set $\beta$ as the cost balance degree, the absolute difference between the total cost of random two vessels less than multiply of the sum of the total cost and the balance of cost degree $\beta$, namely the costs are relatively balanced of random two vessels.

$$
\left|f^{j_{1}}-f^{j_{2}}\right| \leq \beta \cdot\left(f^{j_{1}}+f^{j_{2}}\right) \forall j_{1}, j_{2} \in J .
$$

The integrated berth allocation and quay crane scheduling problem in container terminals can be formulated as follows:

$$
\begin{aligned}
& \min f=\sum_{j \in J} f_{j} . \\
& \text { Subject to: } \\
& \sum_{i \in I} \sum_{k \in K}^{x} \stackrel{x}{=} 1 \quad \forall j \in J \text {. } \\
& \sum_{i j k}^{X} \leq 1 \quad \forall i \in I, k \in K \text {. } \\
& V_{i j}^{j \in J}=\sum_{k \in K}^{X}{ }_{i j k} \quad \forall i \in I, j \in J, k \in K . \\
& J_{i j k}-h_{j} \geq\left(x_{i j k}-1\right) \cdot M \forall i \in I, j \in J, k \in K . \\
& v_{0} \cdot \sum_{i=h_{j}}^{T}{ }_{j t}^{\boldsymbol{\theta}}=E_{j} \quad \forall j \in J . \\
& f_{i j k} \cdot-b_{i j k} \geq\left(x_{i j k}+x_{i j k},-2\right) \cdot M \quad \forall i \in I, j^{\prime} \neq j, k<k^{\prime} \text {. } \\
& v_{i j k}^{i j k}-J_{i j k}=\sum_{i=h_{i}}^{T}{ }_{j t} \quad \forall i \in I, j \in J, k \in K . \\
& \begin{array}{ll}
e_{j}^{-b} \quad v_{i j k} \geq\left(x_{i j k}-1\right) \cdot M & \forall i \in I, j \in J, k \in K . \\
\sum_{i j k}-s_{j} \geq\left(x_{i j k}-1\right) \cdot M & \forall i \in I, j \in J, k \in K .
\end{array}
\end{aligned}
$$

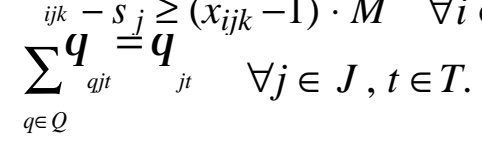

$$
\begin{aligned}
& z_{j t \cdot r_{j}^{\min }} \leq \theta{ }_{j t} \leq z_{j t} \cdot r_{j}^{\max } \quad \forall j \in J, t \in T .
\end{aligned}
$$




$$
\begin{aligned}
& \sum_{q \in Q} \sum_{t=1}^{e_{j}-1} \boldsymbol{\theta}_{q j t}=0 \quad \forall j \in J \\
& \sum \sum_{q j t}^{T} \quad=0 \quad \forall j \in J . \\
& \underset{q \in Q t=e}{Q} \dot{\underline{H}}^{+1} \mathbf{1} \\
& \sum_{j \in J}^{\forall}=1 \quad \forall q \in Q, t \in T \text {. } \\
& \sum_{q \in Q j \in J}^{\theta} \sum_{q j t} \leq Q \quad \forall t \in T \text {. } \\
& f^{j}=\sum_{i \in I} \sum_{k \in K}\left\{C^{1} \cdot z_{j} \cdot\left(b_{i j k}-f_{i j k}\right)+C^{2} \cdot\left(b_{i j k}-f_{i j k}\right)+C^{3} \cdot\left(f_{i j k}-h_{j}\right)\right\} \quad \forall j \in J . \\
& x_{i j k}, \theta_{q j t} \in\{0,1\} \quad \forall i \in I, j \in J, k \in K, q \in Q, t \in T . \\
& b_{i j k}, f_{i j k} \geq 0, \forall i \in I, j \in J, k \in K \text {. }
\end{aligned}
$$

The objective function (2) is to minimize the total cost of vessels. Constraints (3) ensure that each vessel is served in one berth. Constraints (4) enforce that every berth serves up to one vessel at any time. Constraints (5) set up the relationship between $v_{i j}$ and $x_{i j k}$. Constraints (6) assure that the starting time is later than the arrival time. Constraints (7) indicate handling time of vessels is proportion to the total weight. Constraints (8) guarantee that the handling of a vessels starts after the completion of handling its immediate predecessor at the same berth. Constraints (9) set up the relationship of $f_{i j k}$ and $b_{i j k}$. Constraints (10) ensure ending time of a vessel is earlier than the prescriptive leaving time. Constraints (11) ensure starting time of vessel is latter than the prescriptive arriving time. Constraints (12) guarantee that QCs remain the same in the process of the operation.

Constraints (13) implies that the number of assigned QCs of vessel $j \in J$ are in $\left[r_{j}^{\min }, r_{j}^{\max }\right]$.

Constraints (14) and constraints (15) assure that there is no quay crane operating tasks value out of the time window. Constrains (16) ensure that a quay crane at most serves a vessel at each time period. Constraints (17) ensure the number of quay cranes are not more than the total number of quay cranes. Constraints (18) denote the formula of the total cost of random vessel.

\section{Computational Experiments}

Figure 2 shows the relationship of the total cost of vessels with the cost balance degree. A large-scale randomly generated example with 50,80,100 and 120 vessels transported by 9 QCs at 3 berths are adopted in the computational experiments. The available time window of vessels are uniformly distribute in a week. The vessel's total weight of containers is uniformly distribute in $[800,1200]$. The weight of containers is discharged by a QC in an hour is 40t. The handling times of vessels are uniformly distribute in [5,15]. The handling cost, the waiting cost and the QCs operational cost for 1-h is 300,200 and 100, respectively.

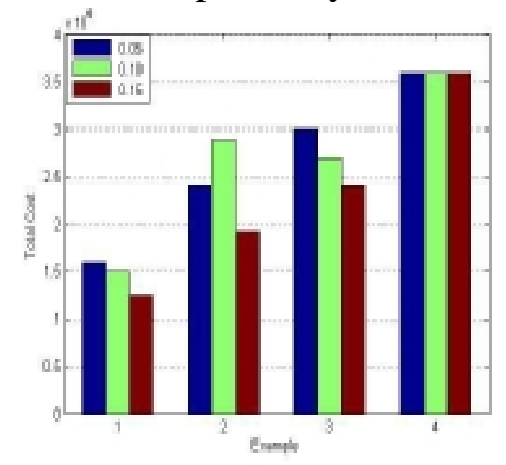

Figure 2: Results of large-scale examples 
The results show that the total cost of vessels is the least with the cost balance degree $\beta=0.15$. So we should consider the influence of cost balance degree in container terminal and choose the reasonable cost balance degree.

\section{Summary and Conclusions}

In this paper, an integer programming model for the integrated berth allocation and quay crane scheduling problem in container terminals is formulated. In this model, some QCs are assigned to transport discharging containers from the vessel. The objective function of this problem is to minimum the total service cost. The total costs include the QCs operation cost, the handling cost and the waiting cost. Compared with previous research, the model formulated in this paper is more practical and comprehensive by considering the cost balance among different vessels in container terminals. By balancing starting time and waiting time, minimum total service costs is achieved.

Due to the NP-hardness of the berth allocation and quay crane scheduling problem, which means that it is impossible to find the optimal solution, a genetic algorithm is designed for the problem solution. Results of large-scale examples show that the proposed heuristic algorithm are effective for solving this problem.

\section{Acknowledgements}

This work is supported by the National Natural Science Foundation of China (Grant No. 71262008), the Scientific Research Foundation for the Returned Overseas Chinese Scholars, State Education Ministry, and Young Talent on Science and Technology Program of University in Inner Mongolia (Grant No. NJYT-13-B02).

\section{References}

[1] K.K. Lai, K. Shih, A study of container berth allocation, Journal of Advanced Transportation. 26 (1992) 75-94.

[2] C.F. Dagnazo, The cranes scheduling program, Transportation Research Part B. 23 (1989) 159175.

[3] A. Imai, E. Nishimura, S. Papadimitriou, The simultaneous berth and quay crane allocation problem, Transportation Research Part E. 44 (2008) 900-920.

[4] M. Rodriguez-Molins, F. Barber, M. Sierre, J. Puente, M. Salido, A genetic algorithm for berth allocation and quay crane assignment, Lecture notes in computer science. Springer, 2012, pp.601610. 